\title{
Methods of linear and nonlinear state estimation of distribution network
}

\author{
Irina Golub ${ }^{1, *}$ and Evgeny Boloev ${ }^{1}$ \\ ${ }^{1}$ Energy Systems Institute of Siberian Branch of the Russian Academy of Sciences, Department of Electric Power System, 664033 \\ Lermontov str., 130, Irkutsk, Russia
}

\begin{abstract}
The paper proposes a new approach to the problem of state estimation of a low voltage distribution network by the measurements coming from smart meters. The problem of nonlinear state estimation based on the measurements of nodal powers and voltages is solved by the method of simple iteration which minimizes the quadratic function of the residues with and without the consideration of the constraint on the zero currents in the transit nodes. The same algorithms are proposed to use for linear state estimation based on the measurements of nodal currents and voltages. The effectiveness of the proposed methods for linear and nonlinear state estimation is illustrated on the 33 nodes three-phase four-wire lowvoltage network.
\end{abstract}

\section{Introduction}

Methods of state estimation (SE) have found wide application in transmission systems designed to transport electrical energy from power plants to distribution networks. The most important event, which contributed to significant improvement of reliability of SE procedure for the transmission system is the introduction of synchronized phasor measurement unit (PMU). Such SE is instrumental in identification of the power passed to the primary medium-voltage distribution network, but it is unable to identify neither overall loads of secondary transformation substations, nor the loads of low voltage distribution network.

Traditional primary and secondary distribution networks operate as radial, with power flows in the feeders oriented from the primary distribution substation towards load nodes. Secondary distribution network is modeled as a three-phase network with neutral wire; its loads can be single-phase, two-phase and three-phase. The return current in neutral wire is due to the unbalanced load and the nonlinear characteristics of electrical equipment through the distribution feeder. The return current may be larger than the phase currents if three-phase loads are seriously unbalanced in some sections [1]. Imaginary components of the voltage drops in the feeders sections virtually equal zero, which is due to a large ratio of resistances to reactance and small lengths of individual sections. Secondary distribution network is modeled without considering mutual reactance between phase wires and without shunt capacitances.

In traditional distribution networks, measurements are usually performed only on primary substations from the medium voltage side, and the state of the secondary distribution substations is unknown. There are methods, in which such measurements are used for approximate estimation of load flow and energy losses in the distribution network [2], but these measurements are not sufficient for SE.

Transition of passive distribution network to active network, is first and foremost related to introduction into it of renewable energy generation sources of stochastic nature, as well as energy storages, charging and discharging of which can be regarded as additional load or generation, active or flexible loads, which allow to transfer the loads of peak period to non-peak within a planned span of time. Directions of power flows in active networks vary during the day, load nodes can become generator nodes, and voltage deviations may exceed admissible values. Voltage control with the help of results of load flow calculation based on the power arriving to the distribution network is impossible even for traditional networks, and even less so for active ones.

Load flow calculation will benefit from the use of average load values calculated on the basis of measurements of energy consumed and registered in the reports of modern automated system of commercial electrical energy accounting in terms of improving accuracy of defining node voltages, but even such accuracy is insufficient to monitor voltages of active distribution networks.

Information of the secondary distribution network state may be obtained by SE methods on the basis of measurements of smart meters (SM), which are installed at load and/or generator nodes. Modern measuring infrastructure Advanced Metering Infrastructure [3] combining an active distribution network with communication network and modern measurement system is responsible for the transfer of SM data to the system of data collection and back. Some SM can measure, besides

\footnotetext{
* Corresponding author: golub@,isem.irk.ru
} 
consumed energy, active and reactive power, active and reactive currents and voltage magnitudes.

Information on such SM measurements is handled by the SE algorithms, the results of which are used to analyze permissibility of voltages and currents, to calculate power losses and indicators of secondary network reliability. In addition, the estimates of power flows and/or currents arriving within each phase from the primary distribution network into the secondary network, as well as the estimates of phase voltages in the secondary network feeding points can be used as loads and voltages for calculation of load flow and/or threephase state estimation of primary distribution network.

A list of tasks, which require information on the loads and load flow of primary distribution network, is extensive. It includes energy loss calculation, regulation of reactive power and voltage, optimization of capacitor placement, network reconfiguration, load forecasting, network strengthening.

However, the process of secondary distribution network SE should be preceded by taking into account the fact that the information on the phase, to which users are connected and for which consumption measuring SM are installed, is generally not available. Knowledge of measurement phase, as well as observability, is an indispensable necessary condition for SE of the secondary distribution network, and, consequently, loads of the primary distribution network.

An effective approach to the definition of the phases is a measurement experiment proposed in [4], which consists in assessment of cross-correlation between voltage profiles in two nodes within certain time period. The first one is the power distribution network node or the closest node with known phases, in each phase measurements are conducted with certain time intervals. The second node is sequentially represented by the nodes with SM, in which measurements of voltage magnitudes are performed at the same time, as the measurements in the phases of the first node. Definition of SM phase connection SM is based on maximum values of crosscorrelation coefficients.

There is no single answer to the question on the possibility of synchronizing SM measurements. The first publications on SM [5] argued that there is a possibility to synchronize SM measurements of currents and voltages in the same distribution network through PLC communication technology, which helps to transmit measurements directly along power transmission lines. However, subsequent publications [6] analyzed the factors impeding synchronization, such as the delay in measurement transfer along communication channels, ranging from a few seconds to several minutes and even up to one day, while measurement upgrade is performed not more often than every 15 minutes.

Despite the existence of SM synchronization methods, such measurements are usually not synchronized and may not be used for SE as synchronized ones, since this would result in unacceptable decrease of estimation accuracy, [7] proposes to compensate for the lack of synchronization with the increase in measurement error with SE up to $10 \%$.

\section{Secondary distribution network state estimation}

Below we analyze two variants of SM measurements in load and/or generator nodes for SE of secondary distribution network: 1) active $z_{P}^{a, b, c}$ and reactive $z_{Q}^{a, b, c}$ node power and voltage magnitudes $z_{U}^{a, b, c}$ in the phases $a, b, c ; 2)$ active $z_{J a}^{a, b, c}$ and reactive $z_{J p}^{a, b, c}$ component of node currents and voltage magnitudes $z_{U}^{a, b, c}$.

Additional measurements are zero values of active and reactive power and active and reactive components of node currents in transit nodes.

Elements of the state vector are represented by real $u_{i}^{\prime a, b, c}$ and imaginary $u_{i}^{\prime a, b, c}$ components of the vectors of phase voltages $u^{a, b, c}=u_{i}^{\prime a, b, c}+j u_{i}^{\prime a, b, c}$ in the nodes $i=1, \ldots, n$ of the design scheme. Measured voltages can be set by their real components, which are equal to the measured voltage magnitudes. The validity of such replacement is related to the proximity of the imaginary voltage components in low-voltage network to zero. Imaginary component of the voltage in the power supply node of the secondary power distribution network is registered as zero.

For the first version of the measurements, it is proposed to use the SE procedure consisting of two iterative repetitive steps. At the first step of the first iteration, the measured values of active and reactive node powers and measurements of voltage magnitudes in the node $i$ are used to determine the values of node currents, which we propose to call pseudo-measurements of node currents

$$
z_{J a i}^{a, b, c}-j z_{J p i}^{a, b, c}=\left(z_{i P}^{a, b, c}-j z_{i Q}^{a, b, c}\right) / z_{U^{\prime} i}^{a, b, c} .
$$

At the second step by pseudo-measurements of currents and real voltage components from the solution of the linear system of measurement equations we define estimation of state variables, which are substituted to the denominator of the expression (1) in subsequent iterations

$$
z_{J a i}^{a, b, c}-j z_{J p i}^{a, b, c}=\left(z_{i P}^{a, b, c}-j z_{i Q}^{a, b, c}\right) /\left(u_{i}^{\prime a, b, c}+j u_{i}^{\prime a, b, c}\right)
$$

Iterative process ends, when the maximum difference between the state variables obtained during adjacent iterations does not exceed predetermined calculation accuracy. Such a procedure for solution of nonlinear systems of measurement equations called simple iteration method is hereby proposed to be applied for secondary distribution network SE instead of traditionally used Newton's iterative method based on linearization of measurement equations through their expansion into Taylor series $[8,9]$. The simple iteration method is known to work well provided the original values of node voltages are appropriate. In the present case, which presumes that SM measure both node powers and voltages, this condition is satisfied. The main advantage of such a solution of nonlinear measurement system is absence of necessity to form Jacobi matrix at iterations, which makes the solution of the nonlinear state estimation task as simple as that of linear task. 
For the second variant of measurements, SE procedure includes solution only linear system equations of measurements of node currents and real voltage components.

Measurement linear equations corresponding to the measured in node $i$ phase values of active and reactive components of the currents and real voltage component can be presented as

$$
\left(\begin{array}{cc}
g_{i}^{a, b, c} & b_{i}^{a, b, c} \\
-b_{i}^{a, b, c} & g_{i}^{a, b, c} \\
\mathrm{I}^{a, b, c} & 0^{a, b, c}
\end{array}\right)\left(\begin{array}{l}
u_{i}^{\prime a, b, c} \\
u_{i}^{\prime a, b, c}
\end{array}\right)=\left(\begin{array}{c}
z_{J a i}^{a, b, c} \\
z_{J p i}^{a, b, c} \\
z_{U^{\prime} i}^{a, b, c}
\end{array}\right)
$$

where $g_{i}^{a, b, c}$ and $b_{i}^{a, b, c}$ are of nodal conductance and susceptance matrices, $\mathrm{I}^{a, b, c}$ and $0^{a, b, c}-$ unit and the zero matrices.

In general terms, the system (3) can be presented as

$$
H_{z}^{a, b, c} \cdot u^{a, b, c}=z_{z}{ }^{a, b, c} .
$$

Similarly, measurement equations for zero node currents in transit node $i$ will appear as

$$
\left(\begin{array}{cc}
g_{i}^{a, b, c} & b_{i}^{a, b, c} \\
-b_{i}^{a, b, c} & g_{i}^{a, b, c}
\end{array}\right)\left(\begin{array}{l}
u_{i}^{\prime a, b, c} \\
u_{i}^{\prime \prime a, b, c}
\end{array}\right)=\left(\begin{array}{l}
z_{J a i}^{a, b, c} \\
z_{J p i}^{a, b, c}
\end{array}\right)=\left(\begin{array}{l}
0 \\
0
\end{array}\right)
$$

or

$$
H_{0}^{a, b, c} \cdot u^{a, b, c}=0 .
$$

We hereby present the combination of equation systems (4) and (6) as

$$
H \cdot u=\left(\begin{array}{c}
H_{z}^{a, b, c} \\
H_{0}^{a, b, c}
\end{array}\right) \cdot u^{a, b, c}=\left(\begin{array}{c}
z_{z}^{a, b, c} \\
0
\end{array}\right)=\bar{z} .
$$

System (6) has a single solution if the number of its equations is equal to the number of variables - basic measurement system, and the rank of matrix $H$ called the matrix of observability $[10,11]$, equals to the number of state variables. To smooth the error impact in individual measurements on the estimation of state variables, measurement equations are multiplied by weight coefficients $R^{-1 / 2}$

$$
R^{-1 / 2} H \cdot u=R^{-1 / 2} \bar{Z},
$$

where $\mathrm{R}$ is measurement error variance.

Such weighing does not affect the solution of the basic measurement equation system. A necessary condition for weighing efficiency is measurement redundancy, which ensures absence of critical measurements, the loss of which leads to a loss of observability. Matrix $R^{-1 / 2} H$ in the presence of redundant measurements becomes redefined, as the number of its lines exceeds the number of columns. There is no classical solution for redefined system (8), but a solution vector $u$ can be acquired, which would help to minimize the distance between the vectors of the right and left parts (8), to this end, the criterion of minimizing the sum of squares differences is used

$$
J(u)=(\bar{z}-H u)^{\mathrm{T}} R^{-1}(\bar{z}-H u) .
$$

This method is called the method of weighted least squares (WLS) and the solution to the task of minimizing can be obtained from the normal system of equations with a square matrix

$$
\left(H^{\mathrm{T}} R^{-1} H\right) u=H^{\mathrm{T}} R^{-1} \bar{Z} .
$$

Estimation of state variables obtained from the solution (10)

$$
u=\left(H^{\mathrm{T}} R^{-1} H\right)^{-1} H^{\mathrm{T}} R^{-1}
$$

is used to calculate estimates of measured variables $z$, in our case - node currents and voltage magnitudes

$$
z=H\left(H^{\mathrm{T}} R^{-1} H\right)^{-1} H^{\mathrm{T}} R^{-1}=H u,
$$

of residues

$$
r=\bar{Z}-\mathrm{z}=\overline{\mathrm{Z}}-\mathrm{Hu} .
$$

Criterion (9) through the weighted residue can be presented as

$$
J(u)=r^{\mathrm{T}} R^{-1} r .
$$

Reduction of weight coefficients for measurements with large residues or their exclusion from the measurements can help to increase the accuracy of estimates of all variables. Another factor affecting estimates is conditionality of matrix $\left(H^{\mathrm{T}} R^{-1} H\right)$ characterizing the impact of measurement errors on the errors of state variables estimation. If the measurements include a large amount of node currents, the conditionality is lower than in the case of measuring line currents. Furthermore, there is an additional factor leading to deterioration of conditionality, which is the need to set very high weight coefficients for equations (6) associated with zero currents.

To obtain a solution ensuring equality to zero of currents in transit nodes, minimization (9) is replaced [12] by Lagrange function minimization, including the function $J_{Z}(u)$ recorded only for the measured variables and constraints of equations (6) with Lagrange multipliers $\lambda$

$$
L=0.5 \cdot\left(\bar{z}-H_{z} u\right)^{\mathrm{T}} R_{z}^{-1}\left(\bar{z}-H_{z} u\right)-\lambda^{\mathrm{T}} H_{o} u .
$$

Solution of linear system of equations with a square matrix is instrumental in determination of state variables estimations $u$

$$
\left(\begin{array}{cc}
H_{z}^{\mathrm{T}} R_{z}^{-1} H_{z} & H_{0}^{\mathrm{T}} \\
H_{0} & 0
\end{array}\right)\left(\begin{array}{l}
u \\
\lambda
\end{array}\right)=\left(\begin{array}{c}
H_{z}^{\mathrm{T}} R_{z}^{-1} z_{z} \\
0
\end{array}\right) .
$$

The solution method in [12] is called WLS method with constraints.

To increase numerical stability, [13] proposes to express $J_{Z}(u)$ in Lagrange function through the residues (14) and, in addition to the constraint (5), to add the constraint on zero equality of the residues (13)

$$
L=0.5 \cdot r^{\mathrm{T}} R_{z}^{-1} r-\lambda^{\mathrm{T}} H_{o} u-\mu^{\mathrm{T}}\left(r-\bar{z}+H_{z} u\right) .
$$

A solution on $u, \lambda$ and $\mu$ in this case may be obtained from the linear system of equations with Hachtel matrix

$$
\left[\begin{array}{ccc}
R_{z} & H_{z} & 0 \\
H_{z}^{\mathrm{T}} & 0 & H_{0}^{\mathrm{T}} \\
0 & H_{0} & 0
\end{array}\right]\left(\begin{array}{l}
\mu \\
u \\
\lambda
\end{array}\right)=\left(\begin{array}{l}
\bar{z} \\
0 \\
0
\end{array}\right) .
$$

Only vector $u$ is used to estimate the state.

It is obvious that the matrices in equations (10), (16), (18) recorded for the three phases are very large, which results in additional computing problems, compared to the case of calculation for single phase with balanced 
loads. The experience of calculating load flow in low voltage four-wire distribution network with unbalanced loads [2] shows that in the case of zero mutual inductances between phase, neutral and earth, calculation of load flow for each phase can be carried out independently. Currents and voltages in a neutral wire and earth can be defined at the second stage of the calculation.

\section{Results of the experiments}

To illustrate the effectiveness of the use of these approaches for state estimation of the three-phase test network the topology data, Fig. 1, hour node powers made up from the loads and renewable generations (photovoltaics) are taken from the work [14]. Only the results of nonlinear state estimation are illustrated on iterations of which a linear task solves using WLS method without constraints, with constraints and Hatchel method.

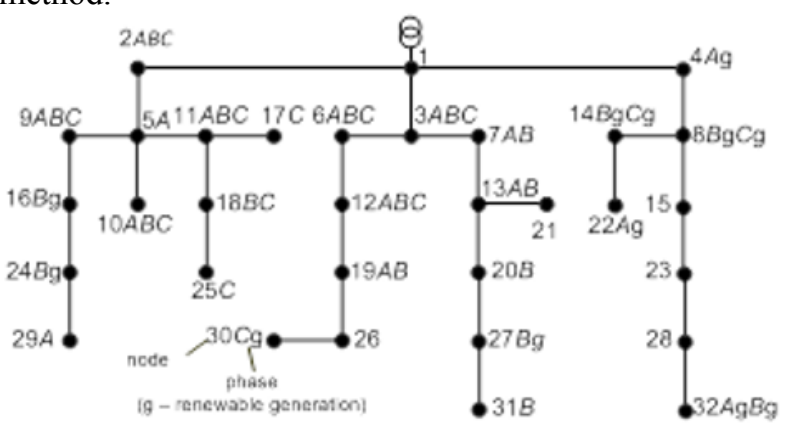

Fig. 1. Scheme of the secondary distribution network

The network includes 32 nodes; the node number 1 is a power supply node. In the phase $A$ there are 15 transit nodes, 12 nodes with the load and 4 nodes with generation. Number of transit, load and generator nodes for phase $B$ is equal to 13,13 and 5 , and for phase $C$ 17,10 and 4.

Information on the loads and the generations was the basis for calculating load flow for each phase of the test network; for impedances of the phase and neural wires, the values, typical for low-voltage distribution networks, were taken. Results of calculation load flow called test results were used for the preparation for measurements of node power and voltage magnitudes including measurement errors. To model errors in measurements, in the test values there were entered errors, standard deviations of which were taken equal to $66 \mathrm{~W}$ and $0.4 \mathrm{~V}$.

The minimum number of measurements providing topological observability of each test network phase is 64 - 32 measurements for the active model and 31 measurement for the reactive model, taking into account zero value of imaginary voltage component fixed in the power supply node of each phase. To ensure observability, SM should be installed in non-transit nodes.

In this case, measurements and pseudomeasurements of 31 active components of nodal currents and real voltage component in one of the nodes of each phase can be considered as base.
The number of redundant measurements in the active model will include real voltage components equal to measurements of voltage magnitudes, minus the voltage measurements included in base composition of measurements. In phase $\mathrm{A}$ there will be 16 redundant measurements, in phase $\mathrm{B}-18$, and in phase $\mathrm{C}-14$. The same measurement redundancy can be also achieved in the reactive model, if the number of redundant measurements at the nodes with the measured voltage magnitudes includes zero measurements of imaginary voltage components, but such approach requires additional research.

Fig. 2 shows daily graphs of absolute values of overall residuals of real voltage components and active node powers in $A, B, C$ phases obtained at the iterations of simple iteration method by WLS methods without constraints -1 , with constraints -2 and by Hachtel method -3 .

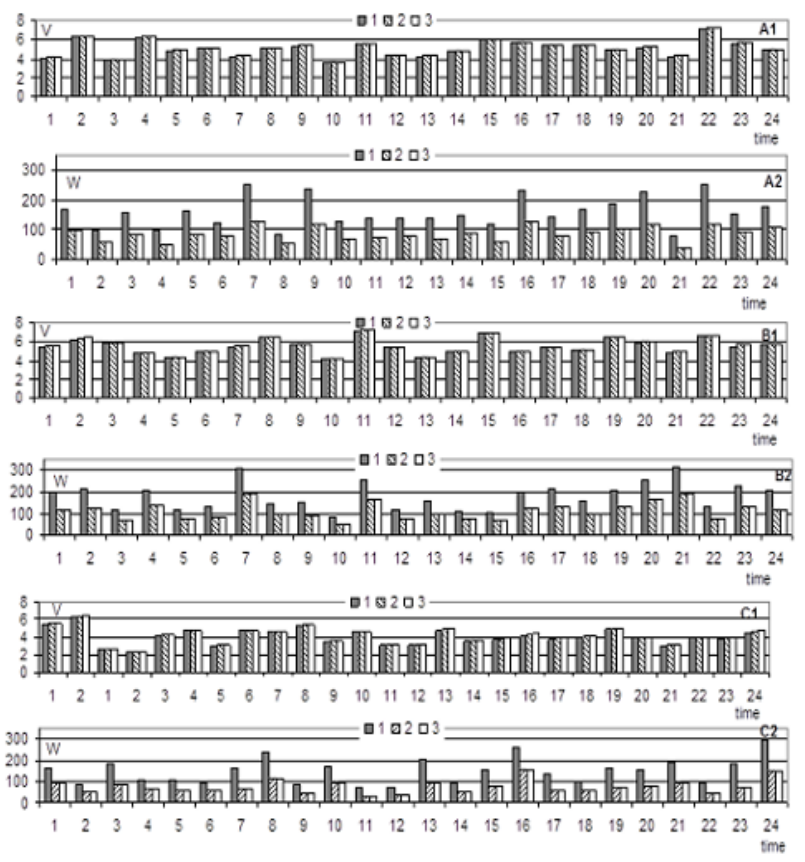

Fig. 2. Total absolute values of the residues in the phases A, B, $\mathrm{C}$ for the real components of the voltages $(\mathrm{A} 1, \mathrm{~B} 1, \mathrm{C} 1)$ and active node powers (A2, B2, C2) obtained by three methods

The analysis of the graphs shows that the residues of voltages are very close for the three methods, which does not make it possible to identify the best method. As regards the residues for active powers, in all phases, for all hours maximum overall are obtained for WLS method without constraints and the same minimum residues - for WLS method with constraints and Hatchel method.

Large residues in the first method appear despite the fact that the weight coefficients associated with zero power injections of transit nodes were included in the measurement equations with larger weights than weights to measured powers.

Let us show that with the gradual increase in weight coefficients for powers of transit nodes from 1 up to 40 times the overall residues for powers will approach the residues obtained by the least squares method with 
constraints, as shown in Fig. 3 for the 15th hour of daily node powers of phase A.

The advantage of using a simple iteration method in comparison with the widely used Newton's method is confirmed by Table 1 which presents the results of calculations for each phase for the 15 th hour of daily node powers.

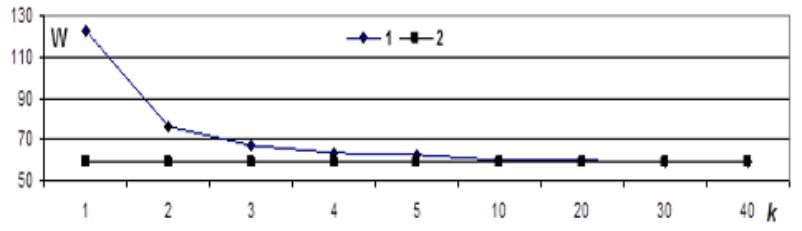

Fig. 3. Change of the total absolute values of nodal powers residues obtained by the WLS method, with an increase in the weight coefficients by $\mathrm{k}$ times for the powers of transit nodes 1 , the residues for the WLS method with constrains -2

Table 1. Comparison methods of solving linear equations systems at the iterations of simple iteration method and Newton's method by the number of iterations (NI), the value of the criterion (VC) of the iteration completion and calculation time (CT)

\begin{tabular}{|c|c|c|c|c|c|c|c|c|c|}
\hline Method & \multicolumn{3}{|c|}{ WLS method without constraints } & \multicolumn{3}{|c|}{ WLS method with constraints } & \multicolumn{3}{|c|}{ Hachtel method } \\
\hline Phase & $\mathbf{A}$ & B & $\mathbf{C}$ & $\mathbf{A}$ & B & $\mathbf{C}$ & $\mathbf{3 A}$ & 3B & $3 \mathrm{C}$ \\
\hline \multicolumn{10}{|c|}{ Simple iteration method } \\
\hline NI & 5 & 5 & 5 & 5 & 4 & 5 & 5 & 4 & 5 \\
\hline $\mathrm{VC}$ & $3,88 \cdot 10^{-13}$ & $6,21 \cdot 10^{-13}$ & $1,52 \cdot 10^{-12}$ & $3,93 \cdot 10^{-13}$ & $6,94 \cdot 10^{-11}$ & $2,99 \cdot 10^{-13}$ & $3,94 \cdot 10^{-13}$ & $6,94 \cdot 10^{-11}$ & $2,98 \cdot 10^{-13}$ \\
\hline $\mathrm{CT}, \mathrm{s}$ & 0,042 & 0,041 & 0,034 & 0,039 & 0,027 & 0,03 & 0,04 & 0,028 & 0,034 \\
\hline \multicolumn{10}{|c|}{ Newton's method } \\
\hline $\mathrm{NI}$ & 7 & 7 & 8 & 7 & 7 & 8 & 7 & 7 & 8 \\
\hline $\mathrm{VC}$ & $4,48 \cdot 10^{-11}$ & $4,85 \cdot 10^{-11}$ & $7,25 \cdot 10^{-12}$ & $4,48 \cdot 10^{-11}$ & $4,83 \cdot 10^{-11}$ & $7,26 \cdot 10^{-12}$ & $4,48 \cdot 10^{-11}$ & $4,83 \cdot 10^{-11}$ & $7,26 \cdot 10^{-12}$ \\
\hline $\mathrm{CT}, \mathrm{s}$ & 0,12 & 0,059 & 0,061 & 0,161 & 0,052 & 0,06 & 0,064 & 0,061 & 0,057 \\
\hline
\end{tabular}

\section{Conclusion}

1. The main result, the efficiency of which was confirmed by calculations, is the use of a simple iteration method to state estimation of the distribution network by SM measuring of nodal powers and voltage magnitudes. 2. The use of the simple iteration method makes the nonlinear state estimation procedure as trivial as the linear state estimation procedure

3. Experimental proof was presented to describe the advantage of using simple iteration method for solving nonlinear equations system as compared to Newton's method by the number of iterations and calculation time, as well as the advantage of using methods of solving measurement linear equations system with constraints for zero currents in transit nodes as compared to method of weighted least squares.

The work is done in the framework of the project III.17.4.2. program of fundamental research SB RAS, registration number AAAA-A17-117030310438-1

\section{References}

1. R.M. Ciric, A.P. Feltrin, L.F. Ochoa IEEE Trans. Power Syst. 18, 1283 (2003)

2. O.N. Voitov, I.I. Golub, L.V. Semenova, Electricity 9 , 38 (2010).

3. M. Emmanuel, R. Rayudu, J NETW COMPUT APPL 74, 133 (2016).

4. H. Pezeshki, P. J. Wolfs, ISGT Europe 3, 1 (2012)

5. S. Santos, A. Llano, A. Arzuaga, T. Arzuaga, L. Marron, M. Zamalloa, CIGRE 44, 8 (2012)

6. A. Alimardani, F. Therrien, D. Atanackovic, J. Jatskevich, E. Vaahedi, IEEE Trans. Smart Grid 6, . 2919 (2015)

7. A. Primadianto, C. Lu, IEEE Trans. Power Syst. 32, 3875 (2017)

8. F.C. Schweppe, J. Wildes, IEEE Trans. Power Appar. Syst. 89, 120 (1970)

9. A.Z. Gamm, Statistical methods of state estimation of electric power systems (Nauka, Moskow, 1976)

10. A.Z. Gamm, I.I. Golub, D.Y. Keselman, Electricity 1, 12 (1975).

11. A.Z. Gamm, I.I. Golub, Observability of electric power systems (Nauka, Moskow, 1990)

12. F. C. Aschmoneit, N.M. Peterson, E.C. Adrian PICA 10, 427 (1977)

13. A. Gjelsvik, S. Aam, L. Holten IEEE Trans. Power Appar. Syst. 104, 2987 (1985)

14. P.C. Olivala, A.G. Madureira, M. Matos Electric Power Systems Research 146, 132 (2017) 